\title{
Detection of Mass in Digital Mammograms
}

\author{
K.Vennila \\ Department of Computer \\ Application \\ Nadar Saraswathi College of \\ Arts \& Science, Theni, India
}

\author{
K.Sivakami \\ Department of Computer \\ Application \\ Nadar Saraswathi College of \\ Arts \& Science, Theni, India
}

\author{
R.Padmapriya \\ Department of Computer \\ Application \\ Nadar Saraswathi College of \\ Arts \& Science, Theni, India
}

\begin{abstract}
Breast Cancer is considered as one of the major causes for the increase in mortality among women. Computerized Mammography helps radiologists as a second reader in diagnosing the abnormalities. This paper aims to detect the masses of the Digital Mammograms. In this approach, the mammogram is subjected to morphological pre-processing, artifacts removal, pectoral muscle segmentation using seeded region growing algorithm. Finally the mass is detected by using Otsu thresholding. The proposed method was tested with Mammographic Image Analysis Society (MIAS) database.
\end{abstract}

\section{Keywords}

Breast Cancer, Pectoral Muscle, Seeded Region Growing, Mass Detection, Otsu thresholding.

\section{INTRODUCTION}

Digital Image Processing has widened its frontiers of applications beyond predictions over the years, due to the advancement of computer technologies and the growing demand for proposing solutions, to the upcoming problems, in the domains such as defense, medicine, remote sensing, criminology, agriculture, IT - enabled services and the like [1],[2].Medical Image Processing has gained significance as it provides feasible technical solutions to supplement/ complement the medical practitioners in prognosis and diagnosis. Breast Cancer is identified as a chronic disease, contributing to female mortality, across the globe [3]. Digital Mammograms have emerged as the best means for prognosis and diagnosis of breast cancer. Research studies reveal that the process of mammogram analysis can be quickened by the intervention of computers. There is a growing demand for accurate and fast computing algorithms to segment the diseased regions from the mammogram, identify the severity of the disease and classify the image components based on the intensity profile of the pixels [4],[5],[6]\&[7].

\section{IMAGE DATASET}

In this work, Mini-MIAS database is used which is freely available for scientific research. The Mammography Image Analysis Society (MIAS), an organization of UK research groups interested in the understanding of mammograms, has produced a digital mammography database. The mammograms in this database were obtained using the mediolateral oblique (MLO) view. The MIAS Database has been reduced to 200 micron pixel edge and clipped/padded so that every image is $1024 \times 1024$ pixels.

\section{METHODOLOGY}

The proposed work consists of three main stages as shown in Fig.1. Mammogram must undergo pre-processing to smooth the image and to remove the artifacts before subjected to the detection of mass in order to produce correct result. This is followed by Otsu thresholding for mass segmentation

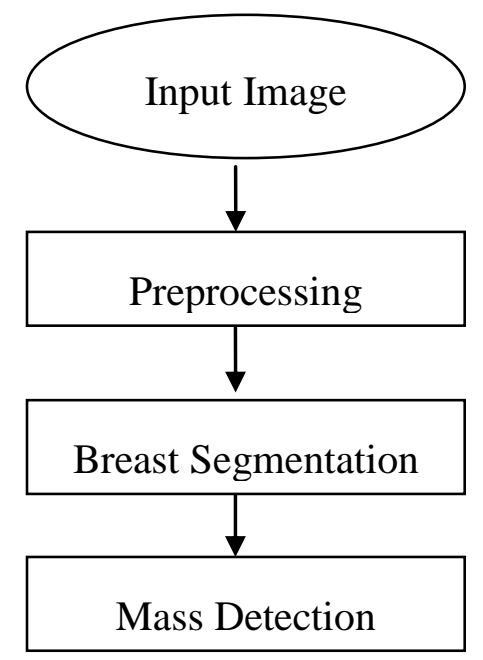

Figure1.The Block diagram of the proposed work.

\subsection{Pre-processing}

It is necessary to improve the quality of the images in order to make the mass detection more reliable. To Input image the structuring element disc is applied with radius 5 starting with dilation followed by erosion, opening, closing and ends with Top hat and Bottom hat transforms. The artifacts are removed by using Matlab Command bwareaopen which keeps the breast portion and removes all the non-breast profile.

\subsection{Breast Segmentation}

This phase involves in removing the pectoral muscle. The pectoral muscle is the brightest region which might produce inaccurate result if not removed. This is done by seeded region growing (SRG) algorithm [8]. The Breast Orientation is determined and the seed pixel is placed in the first pixel in the first row of the image if it is left oriented or else the seed pixel is placed in the last pixel of the first row. The region growing algorithm considers whether the neighboring pixels of seed pixel should be added to the region based on intensity and does this iteratively. 


\subsection{Mass Detection}

In this phase, segmentation of mass is done by one of the best thresholding method called Otsu which maximizes the between-class variance [9].

\section{RESULTS}

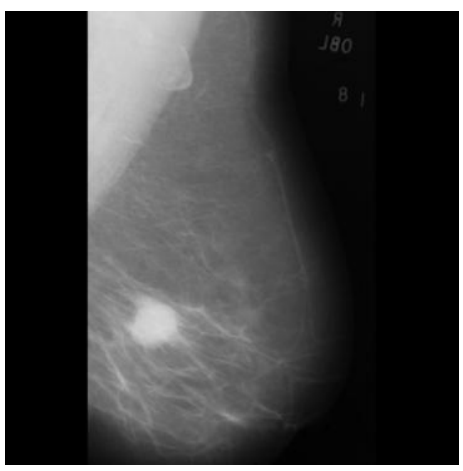

Fig 2: Input Image

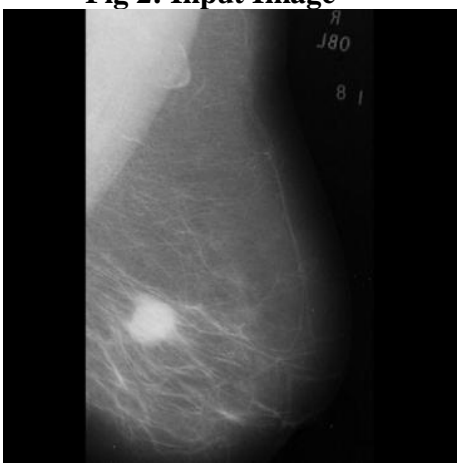

Fig 3: Pre-processed

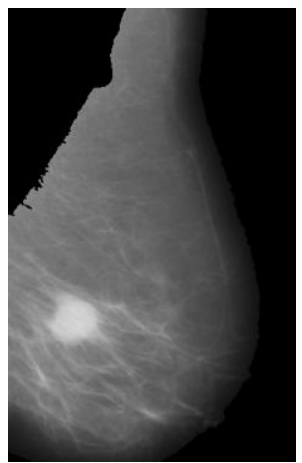

Fig 4: Pectoral Muscle Removed

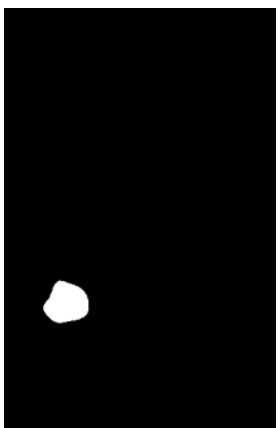

Fig 5: Mass Detected

\section{CONCLUSIONS \& FUTURE WORK}

Computerized Mammography is emerging as one of the most promising approaches that may improve the efficacy. This paper aims at detecting the masses in Digital Mammograms using Otsu Thresholding Method. This work has been implemented and tested using Matlab 7.8. In Future the work has to be revised with a novel approach to achieve above $90 \%$ accuracy in detecting and then classifying the detected mass into benign and malignant.

\section{REFERENCES}

[1] Rafael C Gonzalez, Richard E. Woods, [Digital Image Processing], Second edition, Prentice Hall, (2009).

[2] Milan Sonka, Vaclav Hlavac, Roger Boyle, [Digital Image Processing and Computer Vision], Cengage Learning, (2008).

[3] Preet K.Dhillon, 'Breast Cancer Factsheet',South Asia Network for Chronic Disease, Public Health Foundation of India.

[4] Samir Kumar Bandyopadhyay, 'Survey on Segmentation Methods for Locating Masses in a Mammogram Image', International Journal of Computer Applications (0975 8887)Volume 9- No.11, (2010)

[5] Indra Kanta Maitra, et.al. 'Technique for preprocessing of Digital Mammogram', Computer Methods and Programs in Bio-Medicine 107, pp. 175-188, (2012).

[6] H.D.Cheng Xiaopeng Cai, Siaowei Chen, Liming Hu, Xueling Lou, 'Computer-Aided Detection and Classification of Microcalcifications in Mammograms: A Survey', Pattern Recognition, Vol.36, pp. 2967-2991, (2003).

[7] Claudio Marrocco, Maria Molinara, Ciro D'Elia, Francesco Tortorella, 'A Computer-Aided Detection System for Clustered Microcalcifications', Artificial Intelligence in Medicine, Vol.50, pp.23-32, (2010).

[8] Rolf Adams and Leanne Bischof, 'Seeded Region Growing', IEEE Transactions on Pattern Analysis and Machine Intelligence, Vol.16, No.6 pp. 641-647, (1994).

[9] Ch.Hima Bindu, 'An Improved Medical Image Segmentation Algorithm Using Otsu Method', International Journal of Recent Trends in Engineering, Vol 2, No. 3 pp.88-90, (2009). 